\title{
Handover Incentives for Self-Interested WLANs with Overlapping Coverage
}

\author{
Xenofon Fafoutis, Student Member, IEEE, and Vasilios A. Siris, Member, IEEE
}

\begin{abstract}
We consider an environment where self-interested IEEE 802.11 Wireless Local Area Networks (WLANs) have overlapping coverage, and investigate the incentives that can trigger handovers between the WLANs. Our focus is on the incentives for supporting handovers due solely to the improved performance for all wireless networks. Such incentives arise due to a well-

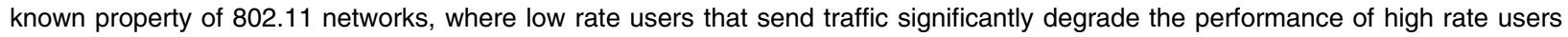
that are associated with the same access point. A key difference of this paper compared to other works is that WLANs are selfinterested, seeking to improve the performance of their own clients. We develop a comprehensive analytical model for accurately identifying and quantifying the handover gains. The model captures cases such as uplink and downlink traffic, wired link capacity constraints, and nonsaturated traffic conditions, and yields a practical handover decision policy. Simulations and experiments on a real testbed verify the accuracy of the model, and indicate that significant gains can be achieved through performance-induced handover incentives.
\end{abstract}

Index Terms-Multirate wireless networks, handovers, incentives

\section{INTRODUCTION}

C ONSIDER an environment where multiple Wireless Local Area Networks (WLANs) have overlapping coverage and operate on the same channel. The WLANs are assumed to be self-interested, meaning that they seek to improve the performance of their clients. The problem we address in this paper is whether there are cases where the selfinterested WLANs have incentives to support handovers, based solely on the improved performance they can achieve for their clients. We develop a comprehensive analytical model to investigate the above problem. Using the model, we can accurately identify the cases where such performance-induced incentives for supporting handovers arise, and quantify the potential improvements for the WLANs involved.

Handover incentives arise due to a well-known property of IEEE 802.11 networks, where the assignment of low and high transmission rate users to the same access point significantly degrades the performance of the high rate users [1]. This occurs because IEEE 802.11's medium access control protocol gives both high and low rate nodes equal chances for accessing the shared wireless channel. However, low rate nodes need more time to send the same amount of data. As a result, when low rate nodes send traffic, high rate nodes suffer significant performance

- X. Fafoutis is with the Department of Informatics and Mathematical Modeling Richard Petersens Plads, Technical University of Denmark, Room 208, Building 322, Lyngby 2800, Denmark.

E-mail:xefa@imm.dtu.dk.

- V.A. Siris is with the Institute of Computer Science, Foundation for Research and Technology-Hellas (FORTH), Heraklion 71110, Greece, and the Department of Informatics, Athens University of Economics and Business, Patission 76, Athens 10434, Greece.

E-mail:vsiris@aueb.gr.

Manuscript received 28 Mar. 2011; revised 5 Sept. 2011; accepted 30 Sept. 2011; published online 20 Oct. 2011.

For information on obtaining reprints of this article, please send e-mail to: tmc@computer.org, and reference IEEECS Log Number TMC-2011-03-0162. Digital Object Identifier no. 10.1109/TMC.2011.230. degradation, achieving throughput equal to that of low rate nodes. There have been a number of works that address the above problem. The handover of nodes that are associated with an access point at a low rate, to another access point with which they can connect at a high rate is one possible solution to the problem; this is the approach taken by works such as [2], [3], [4], which assume cooperation between access points. Alternatively, relays can be used for forwarding traffic that cannot be transmitted directly at high rates [5], [6], [7], [8], [9]. The distinctive feature of the current paper is that we investigate the above problem in the context of self-interested WLANs, which seek to improve the performance of their own clients. Our analytical framework can identify when such handovers can be induced solely by the performance improvements that the self-interested WLANs can achieve. The work in this paper also differs from works such as [10], [11], [12], which deal with the problem of access point and network selection using a game theoretic approach; the key difference is that the above works investigate the problem from the viewpoint of the individual users, namely users seek to maximize their benefit, whereas in our work we consider the gains of WLANs which seek to improve the aggregate performance of their clients, by supporting handovers of clients that belong to other WLANs.

In the case of operator-owned networks, handovers may be supported by cooperation agreements between the operators, which require detailed accounting. On the other hand, our focus is on the incentives for supporting handovers, due solely to the improved performance that handovers yield for both networks without involving any monetary or virtual currency exchange, such as in tokenbased systems [13], [14], [15], or reputation systems [16], [17]. Moreover, similar performance incentives arise in the case of overlapping wireless home networks where cooperation agreements are unrealistic. Although this paper focuses on performance-oriented incentives for triggering handovers, such incentives can also arise in other areas, 


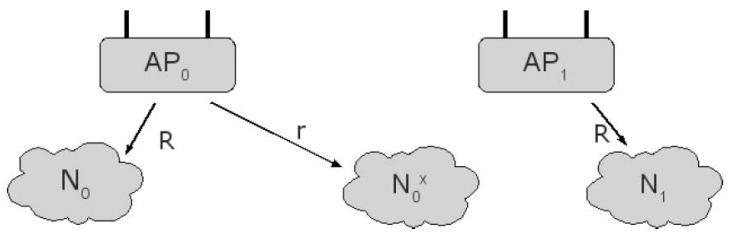

Fig. 1. No handover (case a): $A P_{0}$ serves both high rate $\left(N_{0}\right)$ and low rate $\left(N_{0}^{x}\right)$ nodes, that are clients of $W L A N_{0} . A P_{1}$ serves high rate nodes $\left(N_{1}\right)$ that are clients of $W L A N_{1}$.

such as when forwarding traffic in multihop wireless networks; the key property of wireless networks that can give rise to performance-oriented cooperation incentives is that the transmission rate for different nodes depends on the path attenuation, hence the transmission rate can be different for different nodes.

An assumption about the environment we consider is that two or more WLANs operate on the same channel. Indeed, it is common that there are more than three WLANs within the range of each other [18]. Hence, the number of orthogonal channels available in $802.11 \mathrm{~b} / \mathrm{g}$ or even $802.11 \mathrm{a}$ is not enough to assign different orthogonal channels to the different access points. Moreover, the available nonoverlapping channels will be further reduced as more wireless networks operating in unlicensed bands are deployed and as channel bonding techniques, e.g., in $802.11 n$, are used to increase the bandwidth hence the transmission speeds.

In order to identify and quantify the potential gain of handovers, we propose a comprehensive model that estimates the throughput of each overlapping WLAN in different scenarios. Throughput approximations that account for rate diversity have been used in [19], [20], [21], [22], and [23]. Our modeling framework captures more complicated scenarios, such as cases where the wired connection of access points, rather than the wireless channel, is the bottleneck for the end-to-end throughput. Moreover, our main contribution lies in the application of the above throughput model to predict the impact of handovers and decide whether handing over the nodes that communicate at low rates leads to higher performance for the clients of the overlapping WLANs. The analysis suggests that there are cases where such handovers can yield significant performance improvements. Additionally, based on the necessary conditions for a handover to be beneficial for all overlapping WLANs, we propose a simple policy that can be used by access points in order to decide whether to accept the handover.

In summary, the contributions of the paper are the following:

- We present an analytical model that can approximate the aggregate performance of each WLAN, for a comprehensive set of cases which include the case where the capacity of a wired link constrains the end-to-end throughput, unsaturated traffic conditions, and client differentiation.

- We apply the model to predict when handovers between self-interested WLANs with overlapping coverage are beneficial for all parties, giving all WLANs the incentive to support handovers.

- We present a comprehensive series of numerical evaluations that identify and quantify the performance gains for handovers, we evaluate the proposed
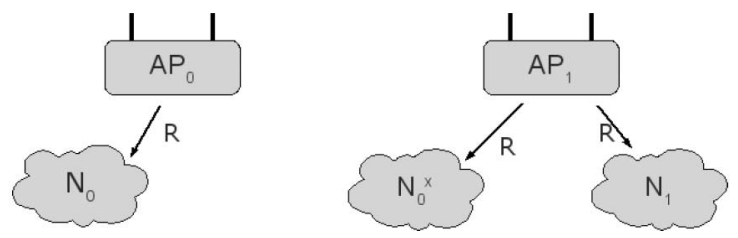

Fig. 2. Handover (case b): $W L A N_{0}$ 's low rate clients $\left(N_{0}^{x}\right)$ are handed over to $A P_{1}$.

model with simulation and testbed experiments, and we discuss the implementation of the proposed handover decision policy in our prototype.

The definition of the incentive problem introduced and analyzed in detail in this paper, to the best of our knowledge, has not appeared in the same form previously in the literature.

The rest of this paper is organized as follows: Section 2 formulates the handover incentives problem, and defines the handover performance gains. Section 3 presents a comprehensive model for estimating the throughput, and the corresponding handover gains; the model includes the case for downlink and uplink traffic, wired link capacity constraints, nonsaturated traffic conditions, and client differentiation. Section 4 describes some variations, such as swapping of low rate clients, multiple low rates, and three access points. Section 5 presents investigations based on the analytical model to identify and quantify the handover gains. Section 6 investigates the accuracy of the model using simulation and testbed experimentation. Section 7 discusses the implementation of the proposed model. Finally, Section 8 concludes the paper.

\section{The Handover Incentives Problem}

In this section, we first discuss the handover incentives problem, and then define the corresponding performance gains that will determine whether the overlapping WLANs have the incentive to support handovers.

Consider two wireless networks, $W L A N_{0}$ and $W L A N_{1}$, containing access points $A P_{0}$ and $A P_{1}$, respectively, Fig. 1. We assume that both access points operate on the same channel and are in the same contention area. The clients of $W L A N_{0}$ include the nodes in sets $N_{0}$ and $N_{0}^{x}$ : the nodes in $N_{0}$ are associated with $A P_{0}$ at a high transmission rate $R$, whereas nodes in $N_{0}^{x}$ are associated with $A P_{0}$ at a low transmission rate $r$. The clients of $W L A N_{1}$ are the nodes in set $N_{1}$, which are associated with $A P_{1}$ at a high transmission rate $R$. For simplicity, we will use the notation $N_{0}, N_{0}^{x}, N_{1}$ to also denote the number of nodes in the corresponding sets.

Due to the low transmission rate of the nodes in $N_{0}^{x}$, and since both APs operate on the same channel, the performance for the clients of both networks is low. The nodes in $N_{0}^{x}$ are closer to $A P_{1}$, hence could associate with this access point at a high transmission rate $R$. WLAN its clients in $N_{0}^{x}$ are handed over to $A P_{1}$, Fig. 2, because the throughput of its clients in both $N_{0}$ and $N_{0}^{x}$ would increase, since there will no longer be low rate nodes. However, whether the performance of $W L A N_{1}$ 's clients $N_{1}$ improves or not if the nodes in $N_{0}^{x}$ are handed over to $A P_{1}$ will depend on two opposite factors: On one hand, there will be a gain because the transmission rate of nodes in $N_{0}^{x}$ will increase. On the other hand, there will be a loss in 
throughput since now the resources of $A P_{1}$ are shared by the clients of $W L A N_{1}\left(N_{1}\right)$ and some clients of $W L A N_{0}$ $\left(N_{0}^{x}\right)$. The magnitude of the two opposite factors will determine whether their combination results in an overall performance improvement or reduction.

Based on the above discussion, we make the following two remarks:

- We consider that the performance of $W L A N_{0}$ includes the performance of nodes in both sets $N_{0}$ and $N_{0}^{x}$, independent of whether the nodes in the latter set are connected to $A P_{0}$ or $A P_{1}$. In other words, the nodes in $N_{0}^{x}$ remain clients of $W L A N_{0}$ even when they are handed over to $A P_{1}$.

- Each WLAN is a self-interested party, seeking to improve the performance of its clients. Hence, a WLAN will support handovers only if they improve the performance of its clients. Moreover, handovers will eventually occur only if both WLANs have the incentive to support them, which occurs if handovers improve the performance of the clients for both WLANs.

The motivation and importance of the above problem becomes clear if we consider the following application scenario: assume two neighboring homes, each with its own WLAN. The users of each home can have a number of wireless stations, which are the clients of that home's WLAN. The problem that we investigate is whether there are incentives, based solely on performance improvements, for the WLAN of one home to support handovers so that its access point serves wireless stations that belong to its neighbor. Handovers will eventually occur only if they improve the performance of the clients for both home networks. Moreover, the performance of each home's WLAN involves the performance of wireless stations belonging to that home, independent of whether they are associated with the home's or a neighbor's access point.

\subsection{Handover Gains}

Next, we define the gains that can arise from handovers. The performance gain of $W L A N_{i}$ is defined as the ratio of the aggregate utility for all clients of $W L A N_{i}$ when a handover occurs, i.e., when the $N_{0}^{x}$ clients are handed over to $A P_{1}$, Fig. 2, over the aggregate utility when there are no handovers, i.e., the $N_{0}^{x}$ clients are associated at a low rate to their home access point $A P_{0}$. When the gain for both WLANs is greater than one, then it is to the advantage of both to support handovers, since they will both increase the performance of their clients; these are the cases we seek to identify, and to quantify the corresponding gains for both WLANs.

For $W L A N_{0}$, the handover gain is given by

$$
\operatorname{Gain}_{W L A N_{0}}=\frac{N_{0} \cdot u\left(X_{N_{0}}^{b}\right)+N_{0}^{x} \cdot u\left(X_{N_{0}^{x}}^{b}\right)}{N_{0} \cdot u\left(X_{N_{0}}^{a}\right)+N_{0}^{x} \cdot u\left(X_{N_{0}^{x}}^{a}\right)},
$$

where the utility $u(\cdot)$ is a function of the achieved throughput. $X_{N_{0}^{x}}^{a}$ and $X_{N_{0}^{x}}^{b}$ are, respectively, the throughput achieved by each of the $N_{0}^{x}$ nodes when there are no handovers and the $N_{0}^{x}$ nodes are associated with $A P_{0}$ at a low rate (case a, Fig. 1), and when there are handovers and these nodes are associated with $A P_{1}$ at a high rate (case $\mathrm{b}$,
Fig. 2). Similarly, $X_{N_{0}}^{a}$ and $X_{N_{0}}^{b}$ are the throughput for each of the $N_{0}$ clients in the case of no handovers and in the case of handovers, respectively.

As discussed above, the $N_{0}^{x}$ nodes are clients of $W L A N_{0}$ even when they are associated with $A P_{1}$. For $W L A N_{1}$, the handover gain is given by

$$
\operatorname{Gain}_{W L A N_{1}}=\frac{u\left(X_{N_{1}}^{b}\right)}{u\left(X_{N_{1}}^{a}\right)},
$$

where $X_{N_{1}}^{a}$ and $X_{N_{1}}^{b}$ are, respectively, the throughput for each of the $N_{1}$ clients of $W L A N_{1}$ in the case of no handovers (case a in Fig. 1) and in the case of handovers (case b in Fig. 2).

In the above discussion, we have assumed that when handovers are performed, all low rate nodes associated with $A P_{0}$ are handed over to $A P_{1}$. In the next section, we prove that if handovers of some low rate nodes provide performance gains, then the gains are maximized if all low rate nodes are handed over to $A P_{1}$.

The utility $u(\cdot)$ is a linear function of the throughput, if the WLANs value the aggregate throughput achieved by their clients. Alternatively, if the utility is a concave function, then more value is given to low throughput clients, compared to high throughput clients.

Based on the estimated gains, $W L A N_{i}$ decides to accept handovers from clients of a neighboring WLAN if its gains are positive, i.e., $\operatorname{Gain}_{W L A N_{i}}>1$. For the scenario in Figs. 1 and 2, which involve two WLANs, handovers will be performed if the gains for both WLANs are positive.

\section{Modeling Framework}

In this section, we begin with the analysis of the scenario discussed in the previous section for long-lived downlink flows with saturated traffic, presenting a model for estimating the throughput per node $X$ that appears in the gain given by (1) and (2). Then, we extend the model for the case where the throughput is limited by the capacity of the wired link that connects an access point to an external network, for unsaturated traffic conditions, for client differentiation, and for the case of uplink traffic.

\subsection{Model for Downlink}

A simple approach for estimating the throughput for the scenario with two access points shown in Fig. 1 is to assume that each access point sends one frame in consecutive rounds. This is a reasonable approximation for long-lived flows with saturated traffic, and assuming that the 802.11's Distributed Coordination Function (DCF) protocol provides long-term fair channel access; the latter is true when all access points are in the same contention area, hence there are no hidden terminals or contention asymmetries.

Case a (no handover). When $N_{0}^{x}$ low rate nodes are assigned to $A P_{0}$, the expected time interval that $A P_{0}$ needs to transmit a frame depends on the percentage of traffic sent to the $N_{0}$ and $N_{0}^{x}$ nodes, since the transmission duration is different due to their different rates. On the other hand, the expected time interval that $A P_{1}$ needs to transmit a frame is the same for all nodes associated with it, since they all have the same rate. We initially assume that $N_{0}, N_{0}^{x}, N_{1}>0$. The long term throughput $X^{a}$ that each access point will 
achieve, assuming both access points transmit frames of the same size, can be approximated by

$$
X^{a}=\frac{p}{\frac{N_{0}}{N_{0}+N_{0}^{x}} T(p, R)+\frac{N_{0}^{x}}{N_{0}+N_{0}^{x}} T(p, r)+T(p, R)},
$$

where $T(p, R)$ is the time for transmitting a packet of size $p$ at rate $R$. The throughput of a node in $N_{0}, N_{0}^{x}$, and $N_{1}$ is then

$$
X_{N_{0}}^{a}=X_{N_{0}^{x}}^{a}=\frac{1}{N_{0}+N_{0}^{x}} X^{a}, \quad X_{N_{1}}^{a}=\frac{1}{N_{1}} X^{a} .
$$

Case $\mathbf{b}$ (handover of low rate users). The low rate nodes $N_{0}^{x}$ are handed over to $A P_{1}$ and all transmissions are at a high rate. Hence, the throughput $X^{b}$ of each access point can be approximated by

$$
X^{b}=\frac{p}{2 \cdot T(p, R)} .
$$

The above holds because all transmissions are at a high rate $R$, hence the packet transmission time is the same for both access points. The throughput of a node in $N_{0}, N_{0}^{x}$, and $N_{1}$ is

$$
X_{N_{0}}^{b}=\frac{1}{N_{0}} X^{b}, \quad X_{N_{0}^{x}}^{b}=X_{N_{1}}^{b}=\frac{1}{N_{0}^{x}+N_{1}} X^{b} .
$$

If we substitute the throughput estimates (3)-(6), in expressions (1) and (2), we can obtain the handover gains for $W L A N_{0}$ and $W L A N_{1}$, respectively. If $N_{0}>0$ and $u(x)=x$, i.e., the WLANs value only the aggregate throughput of their clients, then the above substitution yields the following gains:

$$
\begin{gathered}
\operatorname{Gain}_{W L A N_{0}}=\frac{X^{b}+\frac{N_{0}^{x}}{N_{0}^{x}+N_{1}} X^{b}}{X^{a}}, \\
\operatorname{Gain}_{W L A N_{1}}=\frac{\frac{N_{1}}{N_{0}^{x}+N_{1}} X^{b}}{X^{a}} .
\end{gathered}
$$

The above indicate that handovers are always beneficial for $W L A N_{0}$, i.e., $\operatorname{Gain}_{W L A N_{0}}>1$, since with handovers $W L A N_{0}$ 's clients in $N_{0}^{x}$ obtain resources from $A P_{1}$ hence $W L A N_{0}$ 's clients utilize the channel more than half the time, and $X^{b}>X^{a}$ because there are no low rate nodes.

Unlike $W L A N_{0}$, the performance of $W L A N_{1}$ depends on the following tradeoff: on one hand, the aggregate throughput achieved by $A P_{1}$ increases, i.e., $X^{b}>X^{a}$, due to removing low rate nodes. On the other hand, when nodes in $N_{0}^{x}$ are handed over to $A P_{1}$, the later's resources are now shared by $W L A N_{1}$ 's clients $N_{1}$ and $W L A N_{0}$ 's clients $N_{0}^{x}$. Whether handovers improve the performance of $W L A N_{1}$ depends on the magnitude of the above two factors.

The throughput estimation in (3) and (5) considers the function $T(p, R)$, which denotes the time for transmitting a frame of size $p$, at transmission rate $R$. In Section 5.1, we present an expression for $T(p, R)$ that includes all protocol overheads. For the discussion in this section, we consider the simple expression $T(p, R)=\frac{p}{R}$ which does not account for protocol overheads; this expression captures a key property of wireless networks, namely that the duration of a packet transmission is higher for nodes with a smaller transmission rate. Considering the above simple expression for $T(p, R)$ and (8), the inequality Gain $_{W L A N_{1}}>1$, which indicates when handovers are beneficial for $W L A N_{1}$, is equivalent to

$$
\frac{N_{1}}{N_{0}+N_{0}^{x}}>c, \text { where } \mathrm{c}=\frac{2}{\frac{\mathrm{R}}{\mathrm{r}}-1} .
$$

Hence, to decide whether handovers are beneficial $W L A N_{1}$ simply needs to compare the ratio of its clients and the clients of its neighboring WLAN, with a factor $c$, which depends on the ratio of the high and low transmission rate. Note that the handover acceptance condition (9) for the case of downlink traffic depends only on the total number of $W L A N_{0}$ clients $N_{0}+N_{0}^{x}$, and not separately on the number of high and low rate clients. This in general is not the case for the extensions to the model we present below, where the handover gains depend separately on the number of high and the number of low rate clients. Nevertheless, what is important to note is that for all the extensions, the handover gains can be estimated using simple mathematical formulas that take as input the number of neighboring clients and their respective transmission rates.

As noted above, the handover decision policy for $W L A N_{1}$ given by (9) does not take into account the protocol overheads. Interestingly, the simple form of the inequality in (9) remains the same, even when the protocol overheads, described in Section 5.1, are taken into account.

Up to now we have assumed that when handovers are supported, all low rate nodes are handed over to the visiting access point, $A P_{1}$. The following theorem shows that when there is a gain from handing over some number of low rate nodes, then this gain is maximized if all low rate nodes are handed over. The above has important practical implications. Namely, in order to identify if handovers are beneficial, $W L A N_{1}$ needs to only check if it gains from accepting all low rate clients of $W L A N_{0}$.

Theorem. Assume that $N_{0}, N_{1}>0$ and $N_{0}^{x}>1$. If there are gains for $W L A N_{1}$ when a subset of $W L A N_{0}$ 's low rate clients are handed over to $A P_{1}$, then these gains are maximized if all of $W L A N_{0}$ 's low rate clients $N_{0}^{x}$ are handed over to $A P_{1}$.

The proof of the theorem is given in the Appendix, which can be found on the Computer Society Digital Library at http://doi.ieeecomputersociety.org/10.1109/ TMC.2011.230.

Special case $N_{0}=0$. We now assume that $N_{0}=0$, i.e., there are no clients of $W L A N_{0}$ operating at high rate $R$. Equations (3) and (4) still apply, with $N_{0}=0$.

In the case of handovers, all $W L A N_{0}$ clients will be associated with $A P_{1}$, hence only $A P_{1}$ will be transmitting. Thus, the throughput for $A P_{1}$ can be estimated by

$$
X^{b, N_{0}=0}=\frac{p}{T(p, R)} .
$$

From the last expression, (1), (2), and assuming $u(x)=x$, we obtain the following handover gains:

$$
\begin{gathered}
\operatorname{Gain}_{W L A N_{0}}=\frac{\frac{N_{0}^{x}}{N_{0}^{x}+N_{1}} X^{b, N_{0}=0}}{X^{a, N_{0}=0}}, \\
\operatorname{Gain}_{W L A N_{1}}=\frac{\frac{N_{1}}{N_{0}^{x}+N_{1}} X^{b, N_{0}=0}}{X^{a, N_{0}=0}},
\end{gathered}
$$

where $X^{a, N_{0}=0}$ is given by (3) with $N_{0}=0$. Recall from the above discussion, when $N_{0}>0$ handovers are always 
beneficial to $W L A N_{0}$ because half of the wireless resources are used by the $N_{0}$ clients of $W L A N_{0}$. If $N_{0}=0$, then handovers are not always beneficial for $W L A N_{0}$; whether they are beneficial for $W L A N_{0}$ depends on the share of $A P_{1}$ 's resources that are used by the $N_{0}^{x}$ clients of $W L A N_{0}$ that are handed over to $A P_{1}$, and on the improved throughput achieved by removing low rate nodes.

\subsection{Wired Link Capacity Constraints}

An important application of WLANs is to provide access to a wired network, such as the Internet. It is not uncommon that the wired link connecting a WLAN to an external network is a bottleneck. This can occur for Digital Subscriber Link (DSL) connections, whose speed depends on the distance of the subscriber to the provider's office. Specifically, for distances larger than $3 \mathrm{~km}$, the capacity of Asymmetric Digital Subscriber Link (ADSL+) falls below $8 \mathrm{Mbps}$, which is significantly lower than the maximum throughput of $802.11 \mathrm{a} / \mathrm{g}$. In this section, we extend the throughput model to account for wired capacity constraints. We denote $C_{A P_{0}}, C_{A P_{1}}$ as the capacity of the wired link connecting $A P_{0}, A P_{1}$, respectively, to the external network. In the analysis that follows we consider various relations of $C_{A P_{0}}, C_{A P_{1}}$ and the throughput values $X^{a}, X^{b}$ estimated in the previous section.

Case $C_{A P_{0}} \leq C_{A P_{1}}$. The aggregate throughput of $A P_{0}$ is

$$
X_{C_{A P_{0}}}=\min \left(C_{A P_{0}}, X\right),
$$

where $X$ is the throughput that is estimated by the model in Section 3.1, for either the case of no handovers (3) or handovers (5).

If $C_{A P_{0}}<X$, then $A P_{0}$ uses less than its maximum share of the wireless channel, hence $A P_{1}$ can potentially obtain a larger share. The aggregate throughput of $A P_{1}$ is

$$
X_{C_{A P_{1}}}=\min \left(C_{A P_{1}}, \theta \cdot X_{C_{A P_{0}}}\right),
$$

where factor $\theta \geq 1$ is the ratio of the number of frames sent by $A P_{1}$ over the number sent by $A P_{0}$, in the same time interval. If $A P_{0}$ is not constrained by $C_{A P_{0}}$ then $\theta=1$. On the other hand, if $A P_{0}$ is constrained by $C_{A P_{0}}$, then part of $A P_{0}$ 's share of the medium is available and can be used by $A P_{1}$. When there are no handovers, factor $\theta$ satisfies

$$
X_{C_{A P_{0}}}^{a}=\frac{p}{\frac{N_{0}}{N_{0}+N_{0}^{x}} T(p, R)+\frac{N_{0}^{x}}{N_{0}+N_{0}^{x}} T(p, r)+\theta \cdot T(p, R)},
$$

where $X_{C_{A P_{0}}}^{a}$ is given by (11) for $X \equiv X^{a}$. In the case of handovers, factor $\theta$ satisfies

$$
X_{C_{A P_{0}}}^{b}=\frac{p}{T(p, R)+\theta \cdot T(p, R)},
$$

where as before, $X_{C_{A P_{0}}}^{b}$ is given by (11) with $X \equiv X^{b}$. $X_{C_{A P_{1}}}^{a}, X_{C_{A P_{1}}}^{b}$ can be estimated from (12), where $X_{C_{A P_{0}}}$ is substituted with $X_{C_{A P_{0}}}^{a}$ given by (13), $X_{C_{A P_{0}}}^{b}$ given by (14), respectively.

From the above expressions for $X_{C_{A P_{0}}}^{a}, X_{C_{A P_{0}}}^{b}, X_{C_{A P_{1}}}^{a}, X_{C_{A P_{1}}}^{b}$ (1), (2), and assuming $u(x)=x$, we obtain the following expressions for the handover gains:

$$
\begin{gathered}
\operatorname{Gain}_{W L A N_{0}}=\frac{X_{C_{A P_{0}}}^{b}+\frac{N_{0}^{x}}{N_{0}^{x}+N_{1}} X_{C_{A P_{1}}}^{b}}{X_{C_{A P_{0}}}^{a}}, \\
\operatorname{Gain}_{W L A N_{1}}=\frac{\frac{N_{1}}{N_{0}^{x}+N_{1}} X_{C_{A P_{1}}}^{b}}{X_{C_{A P_{1}}}^{a}} .
\end{gathered}
$$

Case $C_{A P_{0}}>C_{A P_{1}}$. The aggregate throughput of $A P_{1}$ is

$$
X_{C_{A P_{1}}}=\min \left(C_{A P_{1}}, X\right),
$$

where $X$ is the throughput that is estimated by the model in Section 3.1, for either the case of no handovers (3) or handovers (5). The aggregate throughput of $A P_{0}$ is

$$
X_{C_{A P_{0}}}=\min \left(C_{A P_{0}}, \theta \cdot X_{C_{A P_{1}}}\right),
$$

where factor $\theta \geq 1$ is the ratio of the number of frames sent by $A P_{0}$ over the number sent by $A P_{1}$, in the same time interval. When there are no handovers, factor $\theta$ satisfies

$$
X_{C_{A P_{1}}}^{a}=\frac{p}{\theta\left[\frac{N_{0}}{N_{0}+N_{0}^{x}} T(p, R)+\frac{N_{0}^{x}}{N_{0}+N_{0}^{x}} T(p, r)\right]+T(p, R)},
$$

where $X_{C_{A P_{1}}}^{a}$ is given by (15) for $X \equiv X^{a}$. In the case of handovers, all nodes are connected at high rate $R$ and factor $\theta$ satisfies

$$
X_{C_{A P_{1}}}^{b}=\frac{p}{\theta \cdot T(p, R)+T(p, R)},
$$

where as before, $X_{C_{A P_{1}}}^{b}$ is given by (15) with $X \equiv X^{b}$. As above, from the values of $X_{C_{A P_{0}}}$ and $X_{C_{A P_{1}}}$ for the case of no handovers and handovers, using (1) and (2) we can derive the corresponding handover gains.

Case $C_{A P_{0}}, C_{A P_{1}}<X^{a}$, given by (3). In this case, handovers are always beneficial for $W L A N_{0}$, but not beneficial for $W L A N_{1}$. Specifically, in the case of no handovers the aggregate throughput for $W L A N_{0}, W L A N_{1}$ is $C_{A P_{0}}, C_{A P_{1}}$, respectively, whereas in the case of handovers the aggregate throughput for each access point is $C_{A P_{0}}+N_{0}^{x} C_{A P_{1}} /$ $\left(N_{0}^{x}+N_{1}\right), N_{1} C_{A P_{1}} /\left(N_{0}^{x}+N_{1}\right)$, respectively.

Case $C_{A P_{0}}, C_{A P_{1}} \geq X^{b}$, given by (5). In this case, the wired link capacity constraints do not affect handovers, and the gains can be estimated using the model in Section 3.1.

\subsection{Nonsturated Traffic Conditions}

Next, we investigate the case where the flow to one of the nodes is not saturated, and has a maximum sending rate $c_{x}$. As in the previous sections, we assume that traffic flows in the downlink direction. We derive the equations when the unsaturated node is in set $N_{0}^{x}$; a similar approach can be used to derive the equations when the unsaturated node is in set $N_{0}$. Consider $X_{N_{0}^{x}}^{a}$ given by (4) and $X_{N_{0}^{x}}^{b}$ given by (6). We identify the following cases:

Case $X_{N_{0}^{x}}^{a} \leq c_{x}>X_{N_{0}^{x}}^{b}$. In this case, $X^{a}$ and $X^{b}$ are the same as the downlink model in Section 3.1, and are given by (3) and (5), respectively.

Case $c_{x}>X_{N_{0}^{x}}^{a}$. In this case, $X^{b}$ is given by (5). Next, we estimate $X^{a}$.

Let $\mu>1$ be the ratio of the throughput of the saturated node $c_{x}$, over the throughput of an unsaturated node $\frac{X^{a}-c_{x}}{N_{0}+N_{0}^{x}-1}$. Parameter $\mu$ can also be seen as the percentage of 
frames sent to the unsaturated node, over the frames sent to a saturated node in the same time interval. The throughput of $A P_{0}$ and $A P_{1}$ when there are no handovers is

$$
X^{a}=\frac{p}{\frac{N_{0}}{N_{0}+N_{0}^{x}-1+\mu} T(p, R)+\frac{N_{0}^{x}-1+\mu}{N_{0}+N_{0}^{x}-1+\mu} T(p, r)+T(p, R)} .
$$

The handover gains are given by

$$
\begin{gathered}
\operatorname{Gain}_{W L A N_{0}}=\frac{X^{b}+\frac{N_{0}^{x}-1}{N_{0}^{x}-1+N_{1}}\left(X^{b}-c_{x}\right)+c_{x}}{X^{a}}, \\
\operatorname{Gain}_{W L A N_{1}}=\frac{\frac{N_{1}}{N_{0}^{x}-1+N_{1}}\left(X^{b}-c_{x}\right)}{X^{a}} .
\end{gathered}
$$

If there are more than one unsaturated flows with the same rate $c_{x}$, the above expressions can be extended in a straightforward manner by replacing $N_{0}^{x}-1$ with $N_{0}^{x}-n$ and $c_{x}$ with $n \cdot c_{x}$, where $n$ is the number of unsaturated flows. If the flows have a different sending rate, then the extension is more detailed and an iterative procedure such as the one described in [24] applied to the case of single hop flows can be used.

\subsection{Client Differentiation}

The model in the previous subsections assumed that the handed over nodes are treated by $A P_{1}$ identical to the clients of $W L A N_{1}$. From (1) and (2), it is easy to see that the gains for $W L A N_{0}$ are always higher than the gains for $W L A N_{1}$. Hence, $W L A N_{1}$ may wish to differentiate the guest nodes of $A P_{1}$ from its own clients. Such differentiation can be supported by standards such as IEEE 802.11e.

Consider that $A P_{1}$ sends $\eta \geq 1$ frames to the clients of $W L A N_{1}$ for every frame it sends to the guest clients, which belong to $W L A N_{1}$. The handover gains for each WLAN, assuming that each WLAN seeks to improve the aggregate throughput of its clients, are given by the following:

$$
\operatorname{Gain}_{W L A N_{0}}=\frac{X^{b}+\frac{1}{\eta+1} X^{b}}{X^{a}}, \operatorname{Gain}_{W L A N_{1}}=\frac{\frac{\eta}{\eta+1} X^{b}}{X^{a}}
$$

where $X^{a}$ and $X^{b}$ are given by (3) and (5), respectively.

Observe that by increasing $\eta, W L A N_{1}$ can increase the gains it achieves, and simultaneously reduce the gains of $W L A N_{0}$. Moreover, as $\eta \rightarrow \infty$ both WLANs achieve the same gain, equal to $X^{b} / X^{a}$.

If we consider a logarithmic utility $u(x)=\log (x)$, then the gains for $W L A N_{0}$ and $W L A N_{1}$ are the following:

$$
\begin{gathered}
\text { Gain }_{W L A N_{0}}=\frac{N_{0} \log \frac{X^{b}}{N_{0}}+N_{0}^{x} \log \left(\frac{1}{\eta+1} \frac{X^{b}}{N_{0}^{x}}\right)}{\left(N_{0}+N_{0}^{x}\right) \log \frac{X^{a}}{N_{0}+N_{0}^{x}}}, \\
\text { Gain }_{W L A N_{1}}=\frac{\log \left(\frac{\eta}{\eta+1} \frac{X^{b}}{N_{1}}\right)}{\log \frac{X^{a}}{N_{1}}} .
\end{gathered}
$$

As we will see in the numerical investigations of Section 5.3, unlike the case of a linear utility where WLANs value only the aggregate throughput of their clients, in the case of logarithmic utility, for large values of $\eta$, the handover gains for $W L A N_{1}$ can be higher than the gains of $W L A N_{0}$; this occurs because increasing $\eta$ reduces the throughput of $W L A N_{0}$ 's clients that are handed over to $A P_{1}$.

\subsection{Model for Uplink}

In the uplink direction, each node contends for accessing the wireless channel. If we assume fair channel access, then each node transmits one frame in consecutive rounds. Hence, in the case of no handovers the throughput of each node is

$$
X_{N}^{a}=\frac{p}{N_{0} \cdot T(p, R)+N_{0}^{x} \cdot T(p, r)+N_{1} \cdot T(p, R)},
$$

whereas in the case of handover the throughput is

$$
X_{N}^{b}=\frac{p}{N_{0} \cdot T(p, R)+N_{0}^{x} \cdot T(p, R)+N_{1} \cdot T(p, R)} .
$$

From the above equations, assuming that $R>r$, handovers will always be beneficial for both WLANs.

\section{Model Variations}

In this section, we discuss variations of the model presented in the previous section, which include the case when both WLANs have low rate clients which are close to the neighboring access point, when a WLAN has clients with different low rates, and when three access points are in the same area.

\subsection{Swapping Low Rate Nodes}

Next, we assume that both $W L A N_{0}$ and $W L A N_{1}$ have low rate clients, $N_{0}^{x}$ and $N_{1}^{x}$, respectively. These low rate nodes connect at rate $r$ when they are associated with their home access point, but can connect at a high rate $R$ if they are handed over to the neighboring access point.

Case a (no handover). The throughput of each access point is

$$
\begin{aligned}
X^{a}= & \{p\} /\left\{g\left(N_{0}\right) T(p, R)+g\left(N_{0}^{x}\right) T(p, r)+h\left(N_{1}\right) T(p, R)\right. \\
& \left.+h\left(N_{1}^{x}\right) T(p, r)\right\},
\end{aligned}
$$

where $g(x)=x /\left(N_{0}+N_{0}^{x}\right)$ and $h(x)=x /\left(N_{1}+N_{1}^{x}\right)$. The throughput of a node in $N_{0}, N_{0}^{x}$ and $N_{1}, N_{1}^{x}$ is

$$
X_{N_{0}}^{a}=X_{N_{0}^{x}}^{a}=\frac{1}{N_{0}+N_{0}^{x}} X^{a}, \quad X_{N_{1}}^{a}=X_{N_{1}^{x}}^{a}=\frac{1}{N_{1}+N_{1}^{x}} X^{a} .
$$

Case $\mathbf{b}$ (handover of low rate nodes). When low rate nodes are handed over to the neighboring AP, then all nodes operate at a high rate $R$, hence the throughput of each AP is given by (5). The throughput of a node in $N_{0}, N_{0}^{x}$ and $N_{1}, N_{1}^{x}$ is

$$
X_{N_{0}}^{a}=X_{N_{1}^{x}}^{a}=\frac{1}{N_{0}+N_{1}^{x}} X^{b}, \quad X_{N_{1}}^{a}=X_{N_{0}^{x}}^{a}=\frac{1}{N_{1}+N_{0}^{x}} X^{b} .
$$

Finally, the handover gains are

$$
\begin{aligned}
\operatorname{Gain}_{W L A N_{0}} & =\frac{\frac{N_{0}}{N_{0}+N_{1}^{x}} X^{b}+\frac{N_{0}^{x}}{N_{0}^{x}+N_{1}} X^{b}}{X^{a}}, \\
\operatorname{Gain}_{W L A N_{1}} & =\frac{\frac{N_{1}}{N_{0}^{x}+N_{1}} X^{b}+\frac{N_{1}^{x}}{N_{0}+N_{1}^{x}} X^{b}}{X^{a}} .
\end{aligned}
$$




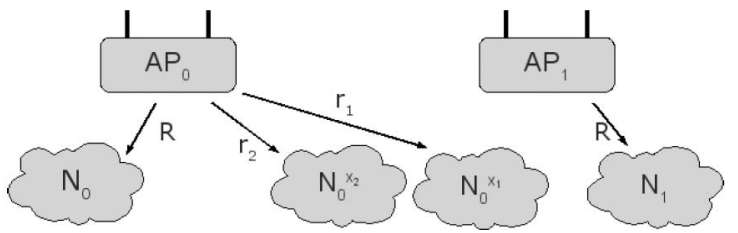

Fig. 3. Mix of low rate nodes. No handovers.

\subsection{Mix of Different Low Rates}

Next, we investigate the case where a mix of different low rate nodes coexist in the same contention area. Specifically, we assume that $W L A N_{0}$ has three sets of clients: clients in $N_{0}$ associate with $A P_{0}$ at a high rate $R$, clients in $N_{0}^{x_{1}}$ associate at a low rate $r_{1}$, and clients in $N_{0}^{x_{2}}$ associate at a low rate $r_{2}$, Fig. 3. We also assume that $r_{1}<r_{2}$.

Case a (no handover). The throughput of each access point is

$$
\begin{aligned}
X^{a}= & \{p\} /\left\{f\left(N_{0}\right) T(p, R)+f\left(N_{0}^{x_{1}}\right) T\left(p, r_{1}\right)\right. \\
& \left.+f\left(N_{0}^{x_{2}}\right) T\left(p, r_{2}\right)+T(p, R)\right\},
\end{aligned}
$$

where $f(x)=x /\left(N_{0}+N_{0}^{x_{1}}+N_{0}^{x_{2}}\right)$. The throughput of a node in $N_{0}, N_{0}^{x_{1}}, N_{0}^{x_{2}}$, and $N_{1}$ is

$$
\begin{aligned}
X_{N_{0}}^{a}= & X_{N_{0}^{x_{1}}}^{a}=X_{N_{0}^{x_{2}}}^{a}=\frac{1}{N_{0}+N_{0}^{x_{1}}+N_{0}^{x_{2}}} X^{a}, \\
& \text { and } X_{N_{1}}^{a}=\frac{1}{N_{1}} X^{a},
\end{aligned}
$$

where $X^{a}$ is estimated from (19).

Case $\mathbf{b}$ (handover of low rate $r_{1}$ and $r_{2}$ nodes). When both low rate $r_{1}$ and $r_{2}$ nodes in $N_{0}^{x_{1}}$ and $N_{0}^{x_{2}}$ are handed over to $A P_{1}$, Fig. 4 , then all nodes transmit at high rate $R$, and the throughput of each access point is given by (5). The throughput of a node in $N_{0}$, and $N_{0}^{x_{1}}, N_{0}^{x_{2}}, N_{1}$ is

$$
\begin{gathered}
X_{N_{0}}^{a}=\frac{1}{N_{0}} X^{b} \text { and } \\
X_{N_{0}^{x_{1}}}^{b}=X_{N_{0}^{x_{2}}}^{b}=X_{N_{1}}^{b}=\frac{1}{N_{1}+N_{0}^{x_{1}}+N_{0}^{x_{2}}} X^{b},
\end{gathered}
$$

where $X^{b}$ is given by (5). The handover gains are

$$
\begin{gathered}
\operatorname{Gain}_{W L A N_{0}}=\frac{X^{b}+\frac{N_{0}^{x_{1}}+N_{0}^{x_{2}}}{N_{0}^{x_{1}}+N_{0}^{x_{2}}+N_{1}} X^{b}}{X^{a}}, \\
\text { Gain }_{W L A N_{1}}=\frac{\frac{N_{1}^{x_{1}}+N_{0}^{x_{2}}+N_{1}}{X^{a}} X^{b}}{X^{a}} .
\end{gathered}
$$

Note that, when $N_{0}>0$, the handover of all low rate nodes is always beneficial for $W L A N_{0}$.
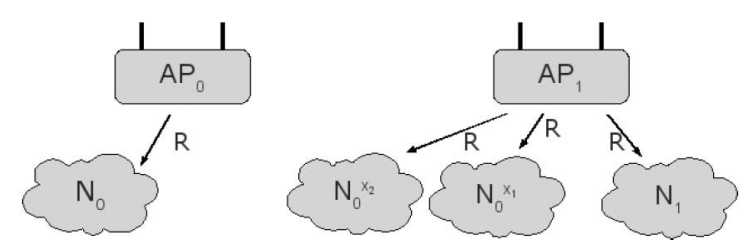

Fig. 4. Mix of low rate nodes. Handover of all $r_{1}$ and $r_{2}$ low rate nodes.

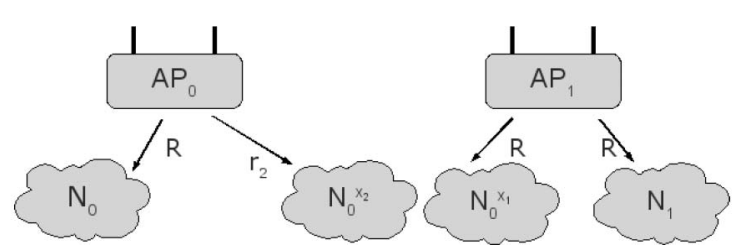

Fig. 5. Mix of low rate nodes. Handover only of $r_{1}$ low rate nodes, $r_{1}>r_{2}$.

Case $\mathbf{b}^{\prime}$ (handover only of low rate $r_{1}$ nodes). When only nodes with the lowest rate $r_{1}$ in $N_{0}^{x_{1}}$ are handed over to $A P_{1}$, Fig. 5, the throughput of each access point is

$$
X^{b}=\frac{p}{\frac{N_{0}}{N_{0}+N_{0}^{x_{2}}} T(p, R)+\frac{N_{0}^{x_{2}}}{N_{0}+N_{0}^{x_{2}}} T\left(p, r_{2}\right)+T(p, R)} .
$$

The throughput of a node in $N_{0}, N_{0}^{x_{2}}$, and $N_{0}^{x_{1}}, N_{1}$ is, respectively.

$$
\begin{aligned}
& X_{N_{0}}^{a}=X_{N_{0}^{x_{2}}}^{b}=\frac{1}{N_{0}+N_{0}^{x_{2}}} X^{b}, \\
& X_{N_{0}^{x_{1}}}^{b}=X_{N_{1}}^{b}=\frac{1}{N_{1}+N_{0}^{x_{1}}} X^{b} .
\end{aligned}
$$

The handover gains are

$$
\begin{gathered}
\operatorname{Gain}_{W L A N_{0}}=\frac{X^{b}+\frac{N_{0}^{x_{2}}}{N_{0}^{x_{2}}+N_{1}} X^{b}}{X^{a}}, \\
\operatorname{Gain}_{W L A N_{1}}=\frac{\frac{N_{1}}{N_{0}^{x_{2}}+N_{1}} X^{b}}{X^{a}} .
\end{gathered}
$$

Note that in the case we have two different low rates, it might be beneficial for both WLANs to hand over nodes with both low rates, or it might be beneficial to hand over only nodes with the lowest rate; this is illustrated in the investigations of Section 5.3. The model presented above can be used to calculate the gains for both cases, and select the best option.

Moreover, although we do not provide a formal proof of a theorem similar to the one in Section 3.1, based on numerical investigations, we conjecture that a similar result holds. Namely, if there are gains in supporting handovers for some subset of low rate nodes with a specific rate, then these gain are maximized if handovers are supported for all low rate nodes with the same rate.

\subsection{Three Access Points}

We now consider the case where three access points, operating on the same channel, are in the same contention area. Nodes $N_{0}^{y}$ that are clients of $W L A N_{0}$ are closer to $A P_{1}$, hence can associate with it at high rate $R$, compared to the rate they associate with $A P_{0}$. Also, nodes $N_{0}^{z}=N_{0}^{x}-N_{0}^{y}$ are closer to $A P_{2}$, hence can associate with it at a high rate $R$. In this scenario, there are three contending transmitters in the downlink, which share the channel in a fair manner.

Case a (no handover). The throughput of each access point is

$$
X^{a}=\frac{p}{\frac{N_{0}}{N_{0}+N_{0}^{x}} T(p, R)+\frac{N_{0}^{x}}{N_{0}+N_{0}^{x}} T(p, r)+2 \cdot T(p, R)} .
$$


Since all clients associated with $A P_{2}$ are served at the same rate $R$, the duration that $A P_{2}$ uses the wireless channel is equal to $T(p, R)$; the same holds for $A P_{1}$. On the other hand, the duration of time that $A P_{0}$ uses the channel depends on the mix of nodes with different transmission rates.

The throughput of a node in $N_{0}, N_{0}^{x}, N_{1}$, and $N_{2}$ is

$$
\begin{gathered}
X_{N_{0}}^{a}=X_{N_{0}^{x}}^{a}=\frac{1}{N_{0}+N_{0}^{x}} X^{a}, \\
X_{N_{1}}^{a}=\frac{1}{N_{1}} X^{a}, \quad X_{N_{2}}^{a}=\frac{1}{N_{2}} X^{a},
\end{gathered}
$$

where $X^{a}$ is estimated by (20).

Case $\mathbf{b}$ (handover of low rate nodes). Now we consider the case where $A P_{1}$ and $A P_{2}$ serve $W L A N_{0}$ 's clients that are close to them. Hence, nodes $N_{0}^{y}$ are handed over to $A P_{1}$ and nodes $N_{0}^{z}$ are handed over to $A P_{2}$. All nodes connect at rate $R$, hence the aggregate throughput of each access point is

$$
X^{b}=\frac{p}{3 \cdot T(p, R)} .
$$

The throughput of a node in $N_{0}, N_{0}^{y}, N_{1}$, and $N_{0}^{z}, N_{2}$ is

$$
\begin{gathered}
X_{N_{0}}^{b}=\frac{1}{N_{0}} X^{b}, \quad X_{N_{0}^{y}}^{b}=X_{N_{1}}^{b}=\frac{1}{N_{0}^{y}+N_{1}} X^{b}, \\
X_{N_{0}^{z}}^{b}=X_{N_{2}}^{b}=\frac{1}{N_{0}^{z}+N_{2}} X^{b},
\end{gathered}
$$

where $X^{b}$ is estimated by (21).

The handover gains are

$$
\begin{gathered}
\operatorname{Gain}_{W L A N_{0}}=\frac{X^{b}+\frac{N_{0}^{y}}{N_{0}^{y}+N_{1}} X^{b}+\frac{N_{0}^{z}}{N_{0}^{z}+N_{2}} X^{b}}{X^{a}}>1, \\
\operatorname{Gain}_{W L A N_{1}}=\frac{\frac{N_{1}}{N_{0}^{y}+N_{1}} X^{b}}{X^{a}}>1, \\
\text { Gain }_{W L A N_{2}}=\frac{\frac{N_{2}}{N_{0}^{z}+N_{2}} X^{b}}{X^{a}}>1 .
\end{gathered}
$$

Note that, as in previous scenarios, when $N_{0}>0$ the handover of all low rate nodes is always beneficial for $W L A N_{0}$.

\section{Analytical InVEStigations}

In this section, we present a series of analytical investigations that aim to identify the performance gains of handovers in different scenarios. First, we describe more accurate estimation of the protocol overhead. Then, we describe the normalized gain metric used to evaluate the models presented in the previous sections. Finally, we present numerical investigations identifying how various factors influence the handover incentives and the corresponding gains. The discussion of the results also shows how the models can be used to provide intuitive and insightful explanations for various behaviors.

\subsection{Protocol Overhead Estimation}

The 802.11 protocol overhead is estimated based on the theoretical maximum throughput [25], which is more accurate than the simple approximation in Section 3.1. We consider the standard DCF protocol without RTS/CTS (Request To Send/Clear To Send). ${ }^{1}$ The time for transmitting one frame consists of five components: $T_{D I F S}, T_{S I F S}, T_{A C K}$, $T_{B O}$, and $T_{D A T A}$. The duration of the DIFS (DCF Interfame Spacing) and Short Interframe Spacing (SIFS) intervals are defined by the standard. $T_{A C K}$ is the time for transmitting an acknowledgment, which in 802.11a is transmitted with the same rate as a data frame, while in $802.11 \mathrm{~b}$ it is transmitted at the minimum rate. $T_{B O}$ is the duration of the backoff, which we discuss below. Finally, $T_{D A T A}$ is the time for transmitting one frame, which includes the Media Access Control (MAC) and physical layer headers, and the frame payload.

Based on the above, we can define $T(p, R)$ that appears in the models of the previous sections as

$$
T(p, R)=T_{D I F S}+T_{S I F S}+T_{A C K}^{R}+T_{D A T A}^{p, R} .
$$

Note that the backoff time $T_{B O}$ is not included in the above expression, since the backoff from multiple frame transmissions are not independent, hence the backoff time is not an additive quantity; this occurs because the backoff counter of multiple contending transmitters decreases simultaneously.

More specifically, when there is a single transmitter, the expected backoff delay is equal to $C W_{\min } / 2$ time slots, where the minimum contention window $C W_{\min }$ and the time slot duration are defined by the standard. When there are multiple contending transmitters, their backoff counter decreases simultaneously, since the backoff counter freezes when a transmission is sensed. Based on the above observation, when there are no collisions, the total backoff delay is independent of the number of contending transmitters, and depends only on the maximum number of frames a single transmitter sends in the time interval over which the throughput is estimated. Hence, the backoff delay can be expressed as $q \cdot T_{B O}^{1}$, where $T_{B O}^{1}=C W_{\min } / 2$ is the average backoff delay when there is only one transmitter, and $q$ is the maximum number of frames sent by a single transmitter, in each transmission round of our approximation. The value of $q$ is 1 for all the equations in Sections 3 and 4, except for (13), (14) where $q=\theta$.

Table 1 shows the values of $T_{D I F S}, T_{S I F S}, T_{B O}^{1}, T_{A C K}$, and $T_{D A T A}$ according to [25]. In all the experiments, we consider payload size $p=1,500$ bytes.

\subsection{Evaluation Metric}

The modeling framework presented in the previous sections estimates the gain that can be achieved when a WLAN accepts handovers. The handover of low rate nodes is performed when all of the involved WLANs improve their performance if they support handovers. In our evaluations, we seek to determine how well such a policy based on the models presented in the previous sections performs. Hence, the evaluation metric should account for both the accuracy of the handover decision and the corresponding gains. Specifically, if we perform $K$ runs, then we define the normalized gain for $W L A N_{i}$ as

$$
\operatorname{NormGain}_{W L A N_{i}}=\frac{1}{K} \sum_{k=1}^{K} x_{i_{k}},
$$

1. The model can easily be extended to the case of RTS/CTS exchange. 
TABLE 1

Delay Components for Transmitting One Frame ( $p$ in Bytes, $R$ in Mbps)

\begin{tabular}{|c||c|c|c|c|c|}
\hline & $T_{D I F S}$ & $T_{S I F S}$ & $T_{B O}^{1}$ & $T_{A C K}$ & $T_{D A T A}$ \\
\hline \hline $802.11 \mathrm{~b}$ & 50 & 10 & 310 & 304 & $192+8 \cdot(34+p) / R$ \\
$802.11 \mathrm{a}$ & 34 & 9 & 67.5 & $20+4\lceil 22+8 \cdot 14 /(4 \cdot R)\rceil$ & $20+4\lceil 22+8 \cdot(34+p) /(4 \cdot R)\rceil$ \\
\hline
\end{tabular}

where $x_{i k}$ is equal to the gain $\operatorname{Gain}_{W L A N_{i}}$ for run $k$ if handovers are performed, and equal to 1 otherwise; hence, the above metric captures both the performance of the handover decision policy and the corresponding handover gains. Moreover, note that handovers are performed only if both WLANs obtain gains hence have the incentives to support them.

\subsection{Investigations}

In this section, we perform a series of analytical investigations to identify the handover gains in different scenarios. The number of clients of each WLAN follows a normal distribution, whose average has default value $\mu=6$. If a WLAN has both high and low rate clients, then the average number for each is $\mu / 2=3$. For all client types, the variance is $\sigma^{2}=2$. Also, we use equation (22) to compute the normalized gain, and consider $K=2,000$ runs for the same scenario.

\subsubsection{Downlink Traffic}

Fig. 6 depicts the normalized gain for $802.11 \mathrm{~b}$ and 802.11a and various values of the low rate $r$, in the case of downlink traffic (Section 3.1). The rate $R$ is equal to the highest rate supported by each protocol, $11 \mathrm{Mbps}$ and $54 \mathrm{Mbps}$ for $802.11 \mathrm{~b}$ and 802.11a, respectively. Both protocols yield similar gains for the two WLANs, and the differences are due to the different overheads. The handover gains for values of $r$ smaller than $2 \mathrm{Mbps}$ and $12 \mathrm{Mbps}$ in $802.11 \mathrm{~b}$ and 802.11a, respectively, are significant. Moreover, observe that the gains for $W L A N_{0}$, which is the WLAN with the low rate clients, Fig. 1, are significantly higher than the gains for $W L A N_{1}$; this occurs because $W L A N_{0}$ 's low rate clients use $W L A N_{1}$ 's resources when they are handed over to $A P_{1}$, Fig. 2.

Fig. 7 depicts the acceptance policy shown in (9) for $802.11 \mathrm{~b}$, where however the factor $c$ is estimated taking into account the more accurate overhead estimation discussed in Section 5.1. The slope of the lines in Fig. 7 corresponds to the value of $c$. All combinations of $N_{0}, N_{0}^{x}, N_{1}$ denoting

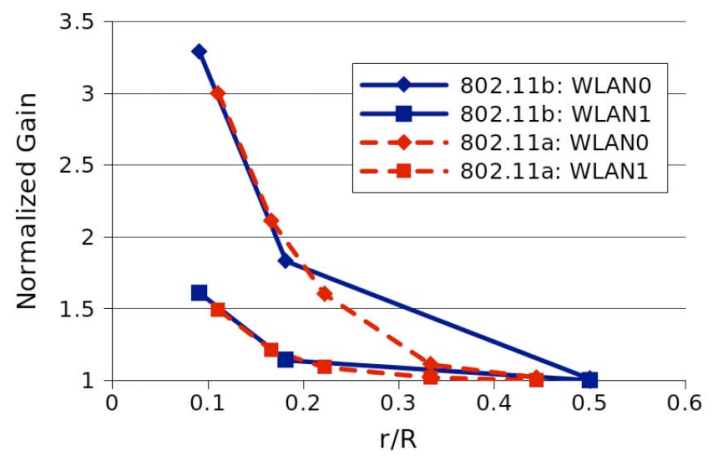

Fig. 6. Downlink traffic in $802.11 \mathrm{a} / \mathrm{b} . W L A N_{0}$ gains more than $W L A N_{1}$ which accepts the handed over nodes. points that are above the line are scenarios where handovers are beneficial for both WLANs. Observe that the number of combinations for which handovers are beneficial becomes smaller, as the low transmission rate $r$ increases.

Fig. 8 shows the normalized gain for $W L A N_{1}$ as a function of the number of $W L A N_{0}$ 's low rate clients that are handed over to $A P_{1}$. Observe that the dependence is not monotonous, and it can happen that the gain initially decreases and then increase as more low rate nodes are handed over to $A P_{1}$; this behavior is a result of two factors: improved performance when the number of low rate nodes is reduced and increased share of $W L A N_{1}$ 's resources used by the handed over nodes. Fig. 8 shows that, for $N_{0}=10, N_{0}^{x}=4, N_{1}=10$, when one or two low rate nodes are handed over to $A P_{1}$ the second factor is more significant, resulting in a reduction of $W L A N_{1}$ 's performance. On the other hand, when three or four nodes are handed over the first factor becomes more significant, resulting in an improvement of $W L A N_{1}$ 's performance.

\subsubsection{Case Where $W L A N_{0}$ Has Only Low Rate Clients}

Fig. 9 shows the normalized gain when $W L A N_{0}$ has only low rate clients, which is the special $N_{0}=0$ considered in Section 3.1. The figures show that the handover gains in this case are significantly higher compared to the case where

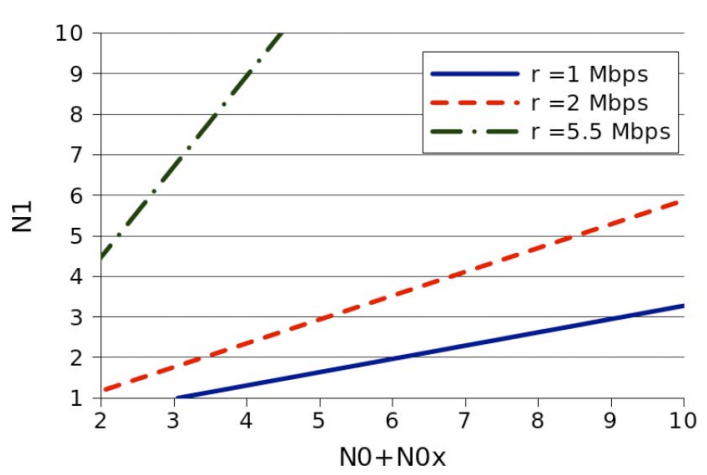

Fig. 7. Acceptance policy for downlink traffic in 802.11 b. Combinations of nodes above the line correspond to scenarios where handovers are beneficial.

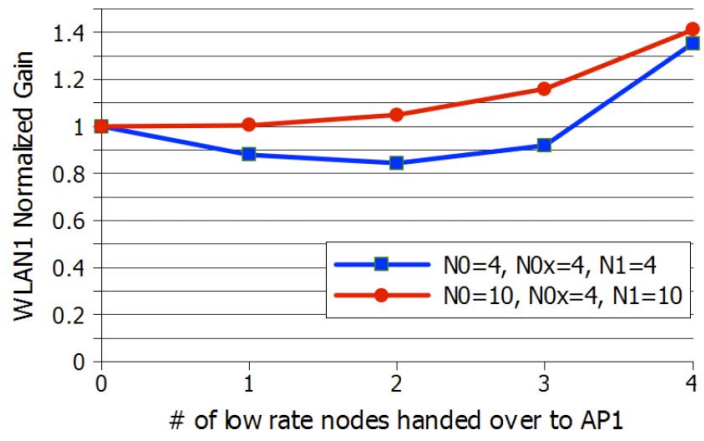

Fig. 8. Normalized gain for $W L A N_{1}$ as a function of the number of $W L A N_{0}$ 's low rate nodes handed over to $A P_{1}$. 


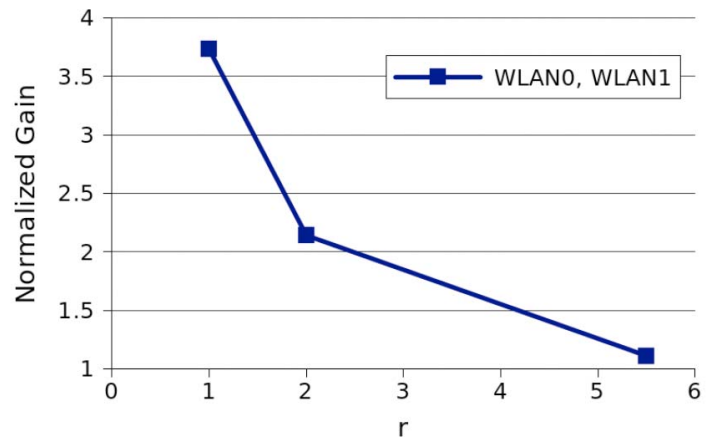

Fig. 9. Case when $W L A N_{0}$ has only low rate clients, downlink traffic, $802.11 \mathrm{~b}$. The gains for both WLANs are the same and significantly higher compared to where $W L A N_{0}$ has both high and low rate clients.

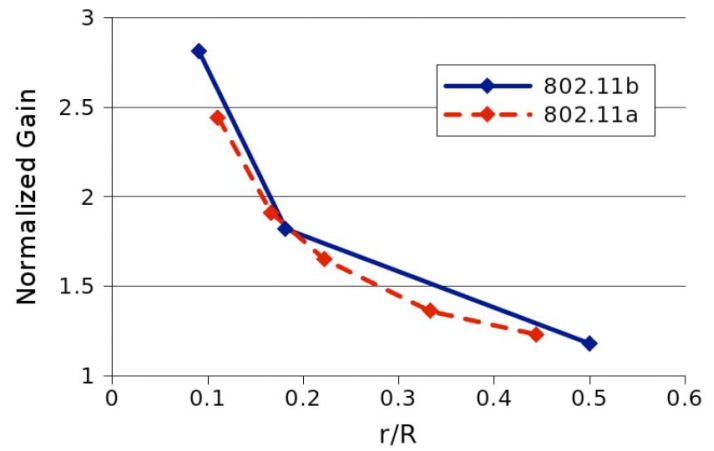

Fig. 10. Uplink traffic in $802.11 \mathrm{a} / \mathrm{b}$. Both WLANs have the same gain.

$W L A N_{0}$ had both high and low rate clients; this occurs because when $W L A N_{0}$ has only low rate clients, all transmissions from $A P_{0}$ involve low rate transmissions, which results in a significant reduction of the throughput for all nodes. Moreover, Fig. 9 shows that the gains for both $W L A N_{0}$ and $W L A N_{1}$ is the same; this occurs because in this case, when handovers are supported, all nodes are served by $A P_{1}$.

\subsubsection{Uplink Traffic}

Fig. 10 shows the normalized gain in the case of uplink traffic (Section 3.5). Unlike the case of downlink traffic, in the uplink case the normalized gain is the same for both WLANs. This occurs because in the uplink direction all nodes contend for the channel and the bandwidth is shared among the nodes associated with both APs. Additionally, note that the normalized gain in the uplink direction is closer to the higher of the two normalized gains (that of $\left.W L A N_{0}\right)$ in the downlink direction.

\subsubsection{Effects of the Node Density}

Next, we investigate how the node density affects the handover gains. In this scenario we consider the case of downlink traffic, and that only $W L A N_{0}$ has low rate nodes with $r=1$ Mbps.

Fig. 11a shows the normalized gain as a function of the average node density, when the variance is $\sigma^{2}=2$. Observe that the normalized gain increases slightly when the average number of nodes is small, and then remains constant. On the other hand, Fig. 11b shows that the normalized gain initially decreases when the variance increases, but remains the same for a variance higher than 10. The above suggest that the handover gains remain significant for different node densities.

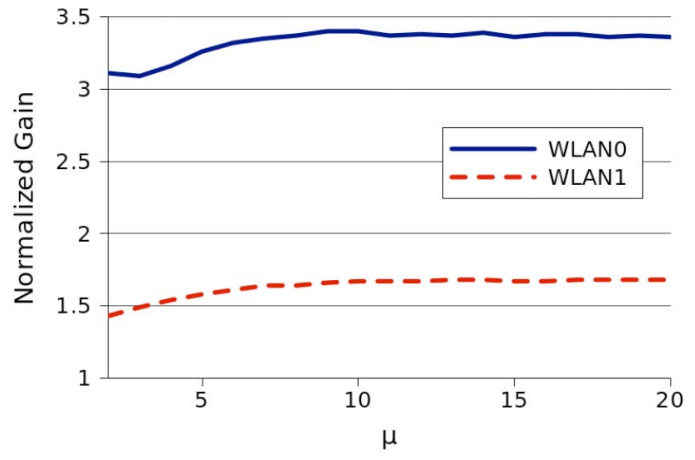

(a) Effects of the average node density. $\sigma^{2}=2$

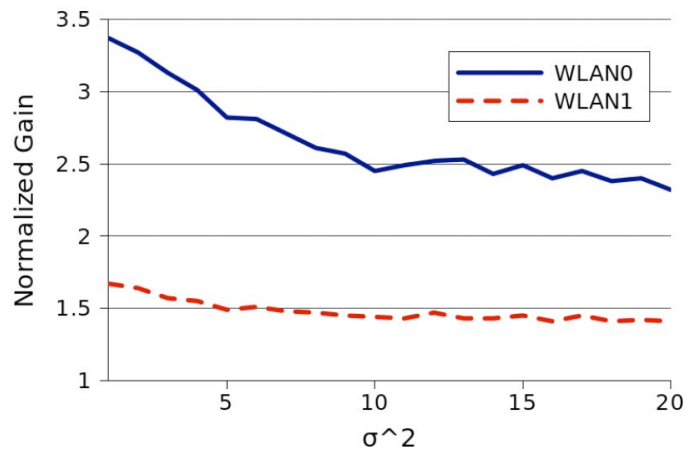

(b) Effect of the variance of the node density. $\mu=6$

Fig. 11. Effect of the node density.

\subsubsection{Wired Capacity Constraints}

Fig. 12a shows the handover gains for different capacity constraints of $A P_{1}^{\prime}$ 's wired connection to an external network, when $A P_{0}$ does not have such a constraint (Section 3.2). When the capacity constraint $C_{A P_{1}}$ is small, then the handover gain for $W L A N_{1}$ is very small. On the other hand, when $C_{A P_{1}}$ is higher than approximately $17 \mathrm{Mbps}$, which is larger than $A P_{1}$ throughput share when there are two contending access points, then the wireless network is the bottleneck, and the wired capacity constraint does not influence the gains. For intermediate values of the capacity constraint $C_{A P_{1}}$, the gain for both $W L A N_{0}$ and $W L A N_{1}$ increases as the constraint increases.

Fig. $12 \mathrm{~b}$ shows the opposite case, where only $A P_{0}$ has capacity constraints. The normalized gain for $W L A N_{0}$ still has a monotonous behavior as $C_{A P_{0}}$ increases. On the other hand, the normalized gain for $W L A N_{1}$ now depends on two factors that have an opposite influence: The first is the increase of the traffic transmitted by $A P_{0}$ as its capacity constraint increases, which results in more low rate traffic transmitted in the network, which in turn makes handovers more beneficial. The second is that as the capacity constraint $C_{A P_{0}}$ increases, then $A P_{1}$ uses a smaller percentage of the wireless resources. The first factor has a larger effect when $C_{A P_{0}}$ is smaller than approximately $9 \mathrm{Mbps}$, whereas the latter factor has a larger effect when $C_{A P_{0}}$ larger than $9 \mathrm{Mbps}$.

\subsubsection{Client Differentiation}

Fig. 13 shows the normalized gain when $A P_{1}$ differentiates $W L A N_{1}$ and $W L A N_{0}$ clients that are handed over, by transmitting $\eta>1$ frames from clients of $W L A N_{1}$ for every frame from clients of $W L A N_{0}$ that are handed over to it (Section 3-D). The results include the case of a linear utility, 


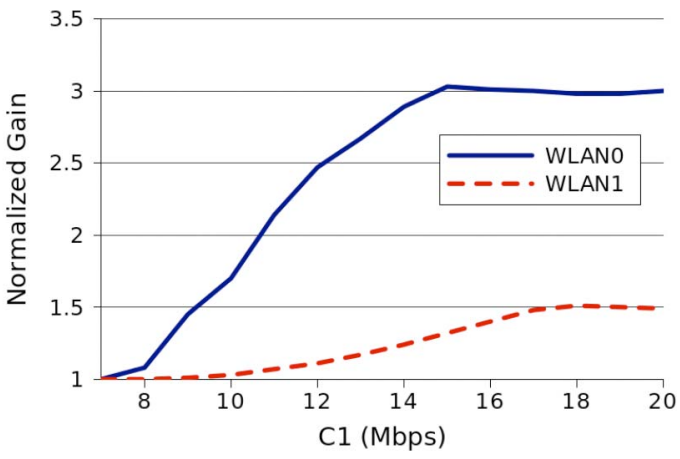

(a) Capacity constraint $C_{A P_{1}}$ for $A P_{1}$.

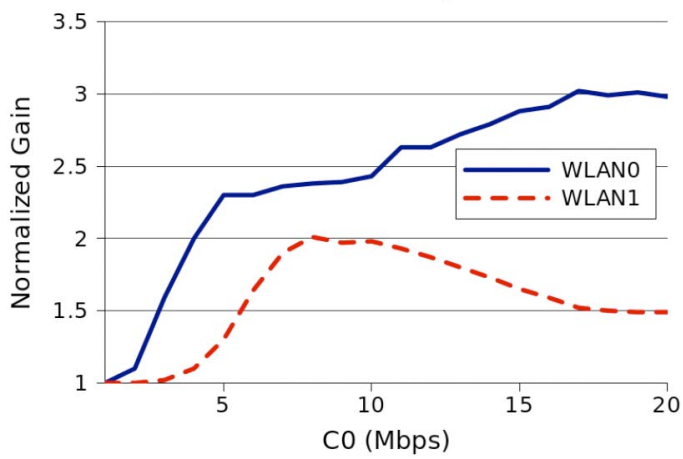

(b) Capacity constraint $C_{A P_{0}}$ for $A P_{0}$.

Fig. 12. Influence of capacity constraints. 802.11a, $r=6$ Mbps.

where the WLANs value the aggregate throughput achieved by their clients, and for the case of a logarithmic utility where more value is given to lower throughputs. As expected, the normalized gain for $W L A N_{1}$ increases as $\eta$ increases, since more traffic from $W L A N_{1}$ clients are transmitted. When the utility is a linear function of the throughput, then as $\eta$ increases, the gains for both WLANs converge to the same value. However, when the utility is a logarithmic function of the throughput, the gains for $W L A N_{1}$ can exceed those of $W L A N_{0}$ as $\eta$ increases; this is because as $\eta$ increases, the throughput achieved by the clients of $W L A N_{0}$ that are handed over to $A P_{1}$ is reduced, resulting in a lower aggregate utility for $W L A N_{0}$, compared to that of $W L A N_{1}$.

\subsubsection{Swapping Low Rate Nodes}

Fig. 14 shows the symmetric scenario where the access points swap low rate nodes (Section 4.1). Comparison of this figure

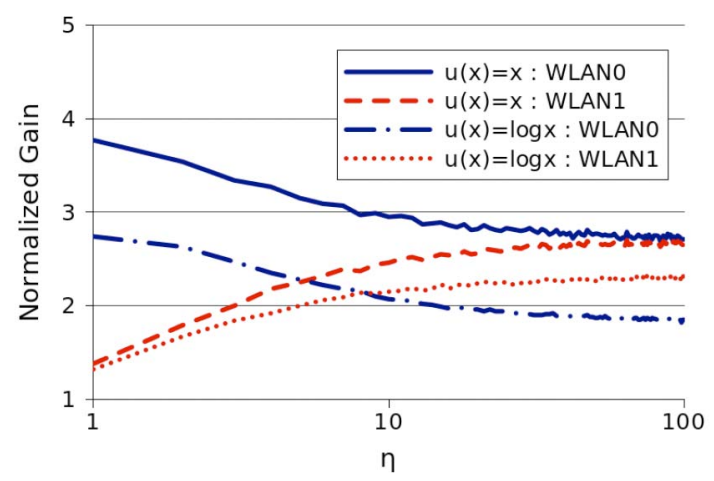

Fig. 13. Client differentiation by $A P_{1}$ in $802.11 \mathrm{~b}$.

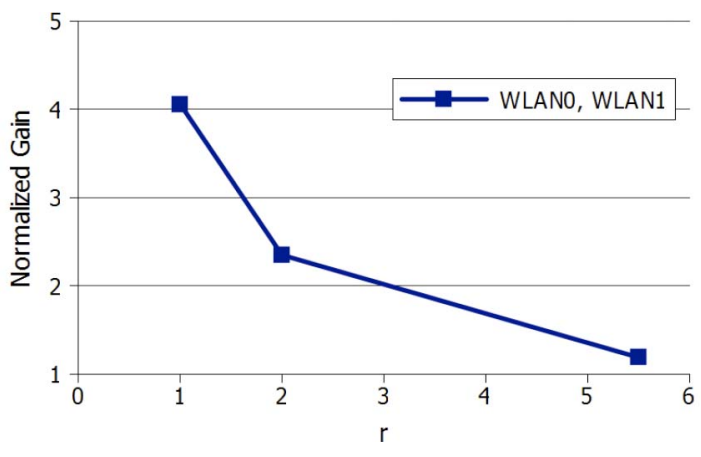

Fig. 14. Swapping low rate nodes. 802.11b.

with Fig. 6 shows that when WLANs swap low rate nodes, the gains they can achieve are higher and more balanced.

\subsubsection{Two Low Rates}

In Fig. 15, we show the gains for the scenario where there are two sets of low rate nodes, with a different rate (Section 4.2). We assume that the nodes in the first set connect at $r_{1}=1 \mathrm{Mbps}$ while those in the other set connect at either $r_{2}=2 \mathrm{Mbps}$ or $r_{2}=5.5 \mathrm{Mbps}$. We investigate whether it is better to hand over all low rate nodes or only the nodes that connect at the lowest rate $r_{1}$. The results shows that it is best for $W L A N_{1}$ is to accept all low rate nodes when $r_{2}=2 \mathrm{Mbps}$, and to accept only the nodes that connect at $r_{1}=1 \mathrm{Mbps}$ when $r_{2}=5.5 \mathrm{Mbps}$. On the other hand, for $W L A N_{0}$ it is always better to hand over all its low rate clients to the neighboring AP.

The above results show that, in the case of multiple low rates, it is not always beneficial to handover nodes with all the different low rates; the model presented in Section 4.2 can be used to decide which low rates are beneficial for an access point to accept.

\subsubsection{Three Access Points}

Fig. 16 depicts the results for the case of three access points (Section 4.3). The performance gains are higher compared to the case of two access points, Fig. 6, since both WLANs now have low rate clients, and benefit from handing over these clients.

\subsubsection{Estimation Error}

The handover decision model presented in Section 3.1 requires estimating the transmission rate with which low rate clients can associate with the visiting access point. Errors in estimating this transmission rate can affect the performance gains. To investigate this influence, we

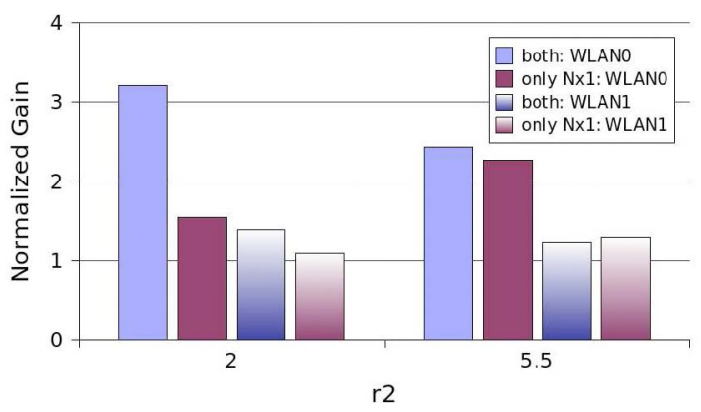

Fig. 15. Mix of low rate nodes where $r_{1}=1 \mathrm{Mbps}$ in $802.11 \mathrm{~b}$. 


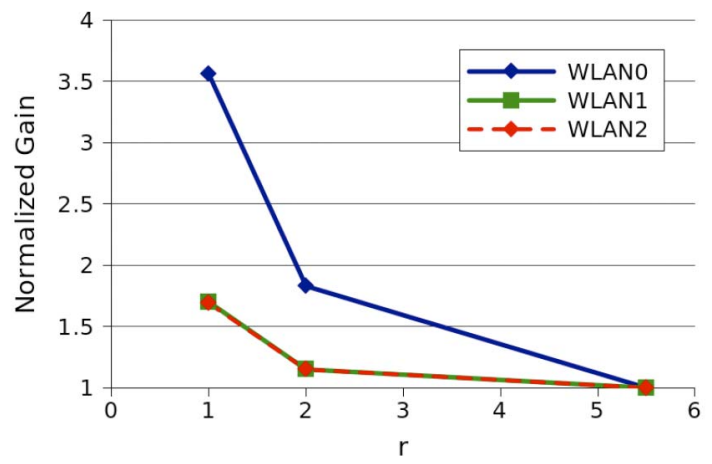

Fig. 16. Three access points in $802.11 \mathrm{~b}$.

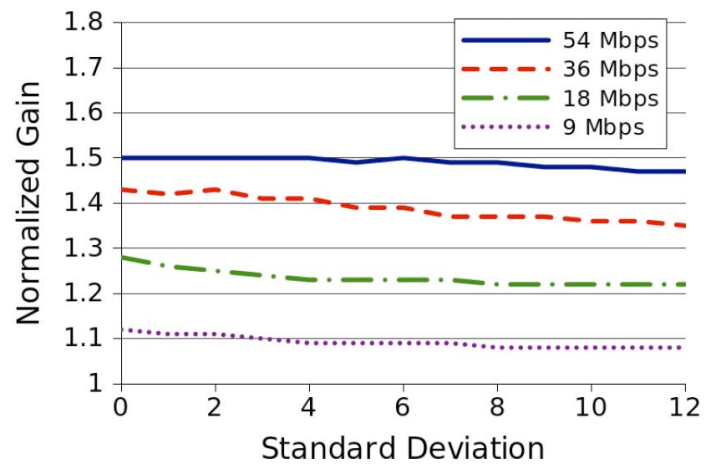

Fig. 17. Gain for $W L A N_{1}$ as a function of the standard deviation of the error in estimating the received signal strength.

consider the scenario in Figs. 1 and 2, where the high rate nodes in $N_{0}$ and $N_{1}$ are associated with access points $A P_{0}$ and $A P_{1}$ at transmission rate $54 \mathrm{Mbps}$, where nodes in $N_{0}^{x}$ are associated with $A P_{0}$ at rate $6 \mathrm{Mbps}$.

Access point $A P_{1}$ estimates the rate at which nodes in $N_{0}^{x}$ can associate with it, by measuring the received signal strength and inferring the corresponding transmission rate; The minimum signal strength to achieve transmission rate $6,9,12,18,24,36,48,54 \mathrm{Mbps}$ is considered to be $-88,-87$, $-85,-84,-83,-80,-76,-71 \mathrm{dBm}$, respectively. Errors are modeled by assuming that the measured signal strength follows a normal distribution, with an average equal to the above minimum signal strength for the corresponding transmission rate, and different values of the standard deviation. Fig. 17 shows how the errors in estimating the received signal strength, hence the errors in estimating the transmission rate with which the nodes in $N_{0}^{x}$ can associate with $A P_{1}$, influence the gain for $W L A N_{1}$. Observe that the average gains show a small dependence on the error in estimating the received signal strength; this is the case because errors in deciding when to support handovers occur when the corresponding gains are small, hence the overall influence on the normalized gain is small.

\section{Evaluation}

In this section, we evaluate the accuracy of the proposed model using simulation and testbed experiments.

\subsection{Simulations}

First, we present simulation results using Network Simulator 2 (NS-2). The default version of NS-2 does not support multiple rates for transmissions between one transmitter

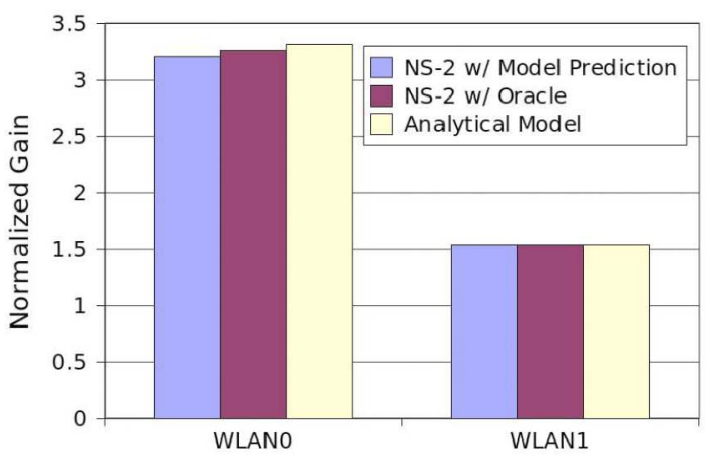

(a) Comparison from NS-2 with handover prediction model, NS-2 with oracle, and analytical model.

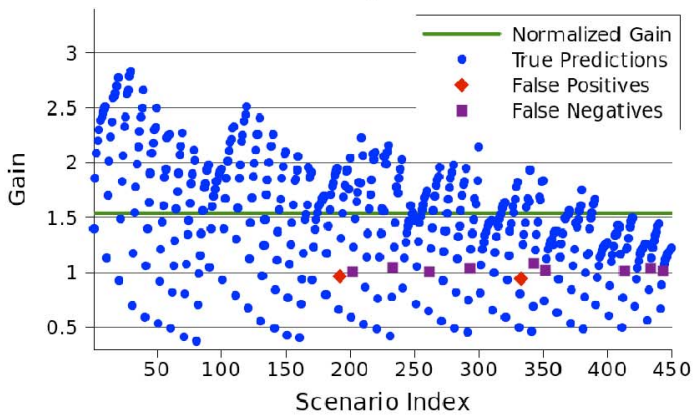

(b) Gain for $W L A N_{1}$, and false positives and false negatives.

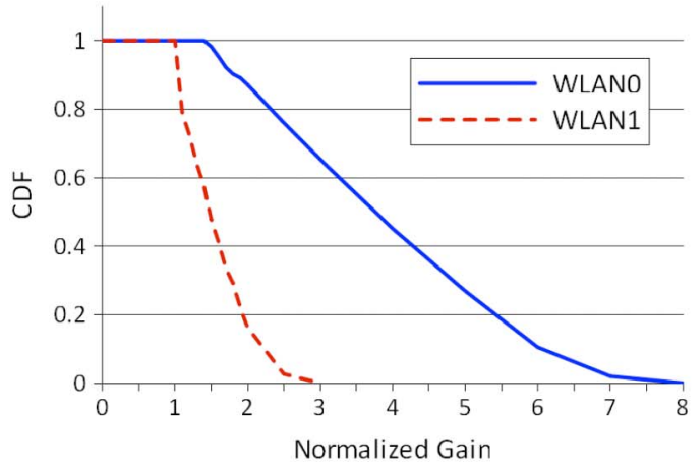

(c) Cumulative distribution function for the handover gains.

Fig. 18. Evaluation of proposed model using simulation.

and many receivers. Hence, we extended NS-2 to support multiple rates by adapting the transmission rate according to the signal strength of the last received frame. We simulate the topology in Figs. 1 and 2 for 802.11b. The low rate $r$ is $1 \mathrm{Mbps}$ and the high rate $R$ is $11 \mathrm{Mbps}$. We performed experiments for a different number of nodes in $N_{0}, N_{0}^{x}$, and $N_{1}$, assuming that each AP serves up to 10 nodes; this resulted in a total 450 scenarios. Fig. 18a compares the normalized gain given by NS-2 when using the handover policy given by (9), where however the factor $c$ is computed using the more accurate protocol overhead estimation discussed in Section 5.1, with the gain given by NS-2 when using an oracle that accurately predicts when a handover is beneficial, and with the analytical model; this figure shows that both the analytical model and the proposed handover acceptance policy achieve performance that is very close to that optimal performance achieved by an oracle, that accurately knows beforehand when handovers are beneficial. Fig. 18b shows the gain for $W L A N_{1}$ for each of the scenarios. This figure also shows that using the 


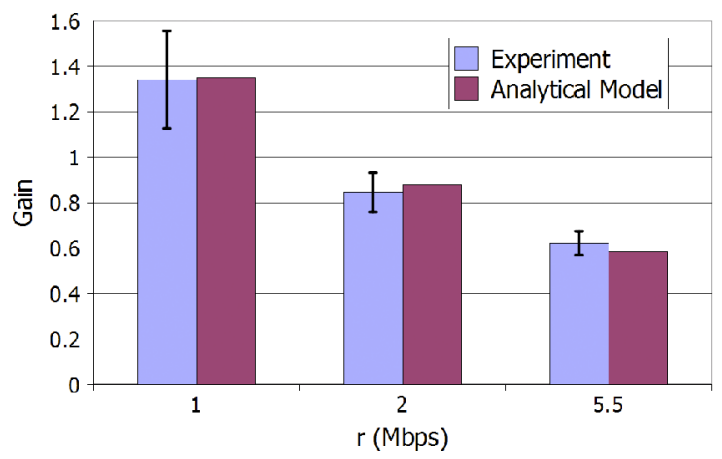

Fig. 19. Comparison of testbed results with analytical model.

proposed model for deciding whether to support handovers results in only 9 false negatives and 2 false positives; moreover, the corresponding gain in the scenarios with a false prediction is very close to one, hence the false predictions have a small influence on the average performance across all scenarios. Finally, Fig. 18c shows the cumulative distribution of the normalized gains for $W L A N_{0}$ and $W L A N_{1}$; note that more than 60 percent of the scenarios yield a gain of more than 230 percent for $W L A N_{0}$, and 50 percent for $W L A N_{1}$.

\subsection{Testbed Experiments}

Next, we evaluate the accuracy of the proposed model using experiments in an actual testbed. We implemented the scenario shown in Figs. 1 and 2, for 802.11b and $N_{0}=N_{0}^{x}=N_{1}=1$; this involved three wireless clients and two Cisco's Aironet 1240AG access points. The low rate $r$ was manually set to 1,2 , and $5.5 \mathrm{Mbps}$, and the high rate $R$ was set to $11 \mathrm{Mbps}$. Fig. 19 compares the average gain of $W L A N_{1}$, for different values of the low rate $r$, measured on the testbed and estimated using the analytical model; the figure shows the average of five runs, and the 90 percent confidence interval. The experimental results show that there is an excellent match between the two. Although the experiments presented above are for a small number of nodes, the scenario considered can be common in practical situations; moreover, in the case of downlink traffic, the access points contend for channel access, hence the number of contending devices (access points) remains the same when the number of end nodes increases.

\section{IMPLEMENTATION}

The handover decision model presented in Sections 2 and 3 requires knowledge of the number of nodes associated with a WLAN's access point, the nodes that are in the same contention area, their transmission rate, and the transmission rate if low rate nodes associate with a visiting access point. The number of nodes, assuming there are no hidden terminals, can be obtained using passive measurements, Fig. 20, by counting the number of unique MAC addresses. The transmission rate to/from nodes can be obtained from 802.11's Physical Layer Convergence Protocol (PLCP) header, when nodes are inside an access point's range. If some nodes are hidden from an access point, then the information for these hidden nodes needs to be communicated, either directly between access points, or through some central

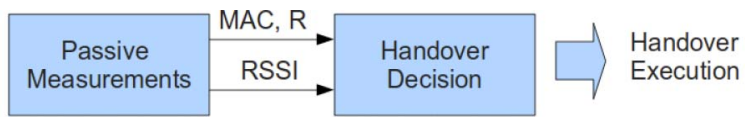

Fig. 20. Handover decision procedure.

server; the latter can be the case, e.g., when home users are subscribers of the same provider, which manages the wireless access points on behalf of its subscribers.

The last parameter that needs to be estimated is the transmission rate if low rate nodes are associated with a visiting access point. This can be estimated by measuring the received signal strength of packets to estimate the corresponding transmission rate. To perform the above passive measurements, an access point can either use a virtual interface or a separate wireless adapter, set to monitoring mode.

Upon identifying that handovers are beneficial, an access point can proceed to allow nearby clients of other WLAN's to associate with it. This can be achieved, without a change at the wireless nodes, by using the same Service Set Identifier (SSID) as the neighboring access point. Moreover, to address security concerns, access to visiting nodes can be offered using a virtual interface at the access point, whereas normal security mechanisms can be used for a WLAN's own clients.

The handover decision procedure in Fig. 20 has been implemented in a prototype, which supports the practicality of the implementation approach described above, and demonstrates $^{2}$ the handover gains presented in the previous section.

\section{Conclusion and Future Work}

The key contribution of the paper is to develop and evaluate a model for identifying and estimating the incentives and gains for supporting handovers when self-interested WLANs coexist in the same area. The self-interested WLANs seek to improve the performance of their clients, hence the incentives for supporting handovers are induced solely by the corresponding performance gains. We have presented results from numerical investigations, simulation investigations, and experiments showing that significant performance gains can be achieved by exploiting the performance-induced incentives for supporting handovers. Finally, our prototype implementation verifies the practicality of the proposed handover decision policy.

An assumption we have made is that access points are in the same contention area. If this does not hold then there can be hidden terminals and contention asymmetries, which for downlink traffic require a more detailed throughput estimation model than the one considered in this paper. Note, however, that for uplink traffic handovers are always beneficial, hence the corresponding handover decision policy is not affected by hidden terminals or contention asymmetries.

Future work involves investigating other environments where performance-induced incentives arise, such as in the case of relaying traffic in multihop wireless networks. A key

2. The prototype implementation was demonstrated at the fifth ACM International Workshop on Wireless Network Testbeds, Experimental evaluation and Characterization (WiNTECH 2010), collocated with ACM Mobicom 2010 
requirement in such an investigation is the development of a simple and accurate model for estimating the end-to-end throughput, while capturing contention asymmetries [26]; initial work in developing such a simple end-to-end throughput model is presented in [24]. Also interesting is to investigate how technologies such as $802.11 \mathrm{n}$, which exploits channel bonding to increase the wireless bandwidth, and cognitive radio, which allows opportunistic access of the wireless spectrum, influence the incentives for cooperation and the corresponding performance gains.

\section{ACKNOWLEDGMENTS}

This work was supported in part by the European Commission in the Seventh Framework Program through project EU-MESH (Enhanced, Ubiquitous, and Dependable Broadband Access using MESH Networks), ICT-215320, http:/ / www.eu-mesh.eu.

\section{REFERENCES}

[1] M.H. Franck, F. Rousseau, G. Berger-Sabbatel, and A. Duda, "Performance Anomaly of 802.11b," Proc. IEEE INFOCOM, 2003.

[2] S. Das, H. Viswanathan, and G. Rittenhouse, "Dynamic Load Balancing through Coordinated Scheduling in Packet Data Systems," Proc. IEEE INFOCOM, 2003.

[3] F. Zdarsky, I. Martinovic, and J. Schmitt, "The Case for Virtualized Wireless Access Networks," Proc. First Int'l Workshop SelfOrganizing Systems, pp. 90-104, 2006.

[4] S. Kandula, K.C.-J. Lin, T. Badirkhanli, and D. Katabi, "FatVAP: Aggregating AP Backhaul Capacity to Maximize Throughput," Proc. Fifth USENIX Symp. Networked Systems Design and Implementation, 2008.

[5] S. Narayanan, P. Liu, and S.S. Panwar, "On the Advantages of Multi-Hop Extentions to the IEEE 802.11 Infrastracture Mode," Proc. IEEE Wireless Comm. and Networking Conf. (WCNC), 2005.

[6] P. Liu, Z. Tao, S. Narayanan, T. Korakis, and S.S. Panwar, "CoopMAC: A Cooperative MAC for Wireless LANs," IEEE J. Selected Areas Comm., vol. 25, no. 2, pp. 340-354, Feb. 2007.

[7] J.-S. Lue and C.-H.R. Lin, "An Opportunistic Relay Method for Increasing Throughput in Multirate IEEE 802.11 Wireless LAN," IEICE Trans. Comm., vol. E88-B, no. 6, pp. 2672-2675, 2005.

[8] L.M. Feeney, B. Cetin, D. Hollos, M. Kubisch, S. Mengesha, and H. Karl, "Multi-Rate Relaying for Performance Improvement in IEEE 802.11 WLANs," Proc. Fifth Int'l Conf. Wired/Wireless Internet Comm. (WWIC), 2007.

[9] P. Bahl, R. Chandra, P.P.C. Lee, V. Misra, J. Padhye, D. Rubenstein, and Y. Yu, "Opportunistic Use of Client Repeaters to Improve Performance of WLANs," Proc. Fourth ACM Int'l Conf. Emerging Networking EXperiments and Technologies (CoNEXT), 2008.

[10] D. Niyato and E. Hossain, "Dynamics of Network Selection in Heterogeneous Wireless Networks: An Evolutionary Game Approach," IEEE Trans. Vehicular Technology, vol. 58, no. 4, pp. 2008-2017, May 2009.

[11] L. Chen, "A Distributed Access Point Selection Algorithm Based on No-Regret Learning for Wireless Access Networks," Proc. IEEE 71st Vehicular Technology Conf. (VTC 2010-Spring), 2010.

[12] A. Argento, M. Cesana, and I. Malanchinii, "On Access Point Association in Wireless Mesh Networks," Proc. IEEE Int'l Symp. World of Wireless Mobile and Multimedia Networks (WoWMoM), 2010.

[13] L. Buttyán and J.-P. Hubaux, "Stimulating Cooperation in SelfOrganizing Mobile Ad Hoc Networks," Mobile Networks and Applications, vol. 8, no. 5, pp. 579-592, 2003.

[14] J. Crowcroft, R. Gibbens, F. Kelly, and S. Östring, "Modelling Incentives for Collaboration in Mobile Ad Hoc Networks," Performance Evaluation, vol. 57, no. 4, pp. 427-439, 2004.

[15] S. Zhong, J. Chen, and R. Yang, "Sprite: A Simple, Cheat-Proof, Credit-Based System for Mobile Ad-Hoc Networks," Proc. IEEE INFOCOM, 2003.

[16] L. Anderegg and S. Eidenbenz, "Ad Hoc-VCG: A Truthful and Cost-Efficient Routing Protocol for Mobile Ad Hoc Networks with Selfish Agents," Proc. ACM MobiCom, 2003.
[17] S. Buchegger and J.-Y.L. Boudec, "Performance Analysis of the CONFIDANT Protocol: Cooperation Of Nodes - Fairness in Dynamic Ad-Hoc NeTworks," Proc. ACM MobiHoc, 2002.

[18] A. Akella, G. Judd, S. Seshan, and P. Steenkiste, "Self-Management in Chaotic Wireless Deployments: Extended Version," Wireless Networks, vol. 13, no. 6, pp. 737-755, Dec. 2007.

[19] A. Kumar, E. Altman, D. Miorandi, and M. Goyal, "New Insights from a Fixed-Point Analysis of Single Cell IEEE 802.11 WLANs," IEEE/ACM Trans. Networking, vol. 15, no. 3, pp. 588-601, June 2007.

[20] A. Kumar and V. Kumar, "Optimal Association of Stations and APs in an IEEE 802.11 WLAN," Proc. Ann. Nat'l Conf. Comm., 2005.

[21] G.S. Kasbekar, J. Kuri, and P. Nuggehalli, "Online Association Policies in IEEE 802.11 WLANs," Proc. Fourth Int'l Symp. Modeling and Optimization in Mobile, Ad Hoc and Wireless Networks, 2006.

[22] B. Kauffmann, F. Baccelli, A. Chaintreau, V. Mhatre, K. Papagiannaki, and C. Diot, "Measurement-Based Self Organization of Interfering 802.11 Wireless Access Networks," Proc. IEEE INFOCOM, 2007.

[23] I. Koukoutsidis and V.A. Siris, "Access Point Assignment Algorithms in WLANs Based on Throughput Objectives," Proc. Sixth Int'l Symp. Modeling and Optimization in Mobile, Ad Hoc and Wireless Network (WiOpt), 2008.

[24] V.A. Siris, G. Stamatakis, and E. Tragos, "A Simple End-to-End Throughput Model for 802.11 Multi-Radio Multi-Rate Wireless Mesh Networks," IEEE Comm. Letters, vol. 15, no. 5, pp. 635-637, June 2011.

[25] J. Jun, P. Peddabachagari, and M.L. Sichitiu, "Theoretical Maximum Throughput of IEEE 802.11 and Its Applications," Proc. IEEE Int'l Symp. Network Computing and Application, 2003.

[26] M. Garetto, T. Salonidis, and E.W. Knightly, "Modeling Per-Flow Throughput and Capturing Starvation in CSMA Multi-Hop Wireless Networks," IEEE/ACM Trans. Networking, vol. 16, no. 4, pp. 864-877, Aug. 2008.

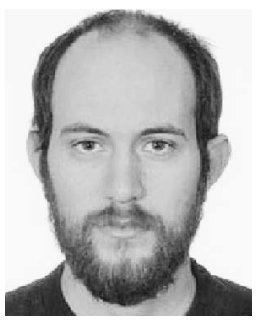

Xenofon Fafoutis received the ptychion (BS) degree from the Department of Informatics and Telecommunications, National and Kapodistrian University of Athens in 2007 and the MS degree from the Computer Science Department, University of Crete, in 2010. Currently, he is working toward the $\mathrm{PhD}$ degree in the Department of Informatics and Mathematical Modeling at the Technical University of Denmark. His current research interests lie in the field of wireless networking and particularly in energy harvesting wireless sensor networks. He was also associated with the Institute of Computer Science of the Foundation for Research and Technology-Hellas (FORTH). He is a student member of the IEEE.

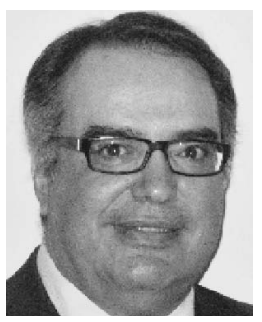

Vasilios A. Siris received a degree in physics from the National and Kapodistrian University of Athens, Greece, in 1990, the MS degree in computer science from Northeastern University, Boston, in 1992, and the PhD degree in computer science from the University of Crete, Greece, in 1998. He has been working as an assistant professor in the Department of Informatics, Athens University of Economics and Business, since 2009, and as a research associate at the Institute of Computer Science of FORTH. From 2002 to 2008, he was an assistant professor at the University of Crete. In Spring 2001, he was a visiting researcher at the Statistical Laboratory of the University of Cambridge, and in Summer 2001 and 2006, he was a research fellow at the research laboratories of British Telecommunications (BT), United Kingdom. His current research interests include resource management and traffic control in wired and wireless networks, traffic measurement and analysis for monitoring quality of service and intrusion/anomaly detection, and architecture of mobile communication systems and future networks. He has served as the general chair or technical program chair for various international conferences and workshops, such as Wired/Wireless Internet Communications 2008, IEEE WoWMoM 2009, HotMESH 2011, and IEEE Broadband Wireless Access 2011. He is currently on the editorial board of the Computer Communications Journal. He is/was the principal investigator and coordinator for many research and development projects funded by the European Commission, the Greek government, and industry. $\mathrm{He}$ is a member of the IEEE. 